\title{
Nonsaturable absorption in alumino-silicate bismuth-doped fibers
}

Cite as: J. Appl. Phys. 109, 023113 (2011); https://doi.org/10.1063/1.3532049

Submitted: 24 June 2010 . Accepted: 02 December 2010 . Published Online: 20 January 2011

A. V. Kir'yanov, V. V. Dvoyrin, V. M. Mashinsky, Yu. O. Barmenkov, and E. M. Dianov

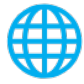

\section{ARTICLES YOU MAY BE INTERESTED IN}

Efficient all-fiber bismuth-doped laser

Applied Physics Letters 90, 031103 (2007); https://doi.org/10.1063/1.2431762

Enhanced broadband near-IR luminescence and gain spectra of bismuth/erbium co-doped fiber by 830 and $980 \mathrm{~nm}$ dual pumping

AIP Advances 7, 045012 (2017); https://doi.org/10.1063/1.4981903

Clustering in erbium-doped silica glass fibers analyzed using $980 \mathrm{~nm}$ excited-state absorption Journal of Applied Physics 76, 4472 (1994); https://doi.org/10.1063/1.357278 


\title{
Nonsaturable absorption in alumino-silicate bismuth-doped fibers
}

\author{
A. V. Kir'yanov, ${ }^{1,2, a)}$ V. V. Dvoyrin, ${ }^{3}$ V. M. Mashinsky, ${ }^{3}$ Yu. O. Barmenkov, ${ }^{1}$ and \\ E. M. Dianov ${ }^{3}$ \\ ${ }^{1}$ Centro de Investigaciones en Optica, Loma del Bosque 115, Col. Lomas del Campestre, \\ Leon 37150 Guanajuato, Mexico \\ ${ }^{2}$ A. M. Prokhorov General Physics Institute (RAS), Vavilov Str. 38, Moscow 119991, Russian Federation \\ ${ }^{3}$ Fiber Optics Research Center (RAS), Vavilov Str. 38, Moscow 119333, Russian Federation
}

(Received 24 June 2010; accepted 2 December 2010; published online 20 January 2011)

\begin{abstract}
We report an experimental and theoretical investigation of fluorescence decay and transmission coefficient at $\sim 1 \mu \mathrm{m}$ pumping for a series of bismuth $(\mathrm{Bi})$ doped alumino-silicate fibers with different concentrations of $\mathrm{Bi}$ centers. By modeling the experimental data, we show that the excited-state absorption (ESA) and up-conversion (UC) processes are responsible for a growth of nonsaturable absorption and deviation from exponential the fluorescence decay in the fibers with an increase in $\mathrm{Bi}$ centers content. Assuming that $\mathrm{Bi}$ centers ensemble is composed of subsystems of single and paired centers, and an increase in partial weight of the latter with a $\mathrm{Bi}$ centers concentration growth, we can successfully explain the experimental data. Also, these assumptions allow us to estimate the constants characterizing the ESA and both homogeneous and inhomogeneous UC processes in the fibers. (C) 2011 American Institute of Physics.
\end{abstract}

[doi:10.1063/1.3532049]

\section{INTRODUCTION}

Bismuth (Bi) doped fibers with alumino-silicate host have been of increasing interest in the past few years as an active medium for amplifying and lasing aimed on the spectral region $1.15-1.3 \mu \mathrm{m}$ when pumped at $\sim 1 \mu \mathrm{m}$ wavelength. ${ }^{1-6}$ Bi-doped fibers have been as well shown to be an effective passive Q-switch element when placed in the cavity of an Yb-doped fiber laser (YFL). ${ }^{7}$ In spite of remarkable success in the field, there remain certain obstacles for further efficiency enhancement of Bi-doped fiber lasers and amplifiers because of a lack of understanding of the nature of Bi centers. ${ }^{8-19}$

In the present work, we report a study of the two crossrelated characteristics, the near infrared (NIR) fluorescence decay and the nonlinear (relatively to the incident power) transmission coefficient, both obtained at $\sim 1 \mu \mathrm{m}$ excitation for a series of Bi-doped fibers with a substantially different $\mathrm{Bi}$ content. In the series, small-signal absorption (SSA) coefficient of $\mathrm{Bi}$ centers is varied in the range from tenths to tens decibels per meter, allowing inspection of the nonlinearities arising due to the interaction and clustering effects. These effects, being a background of up-conversion (UC), together with excited state absorption (ESA), seems to be a reason for strong limitation in efficiency of Bi-doped fiber lasers and amplifiers at pumping within the $\sim 1 \mu \mathrm{m}$ absorption band of Bi centers. ${ }^{20-22}$ Although the ESA and homogeneous UC (HUC) effects in Bi-doped fibers were already attempted to be addressed, ${ }^{23-27}$ the inhomogeneous UC (IUC), or a clustering effect, remained, to the best of our knowledge, out of scope in previous studies, except of our paper. $^{25}$ In the present report, we provide the reader with

${ }^{a)}$ Electronic mail: kiryanov@cio.mx. further experimental information on these phenomena in $\mathrm{Bi}$ doped alumino-silicate fibers and their theoretical modeling.

\section{EXPERIMENTAL}

All the Bi-doped fibers we dealt with in experiments were drawn from alumino-silicate host preforms fabricated by the MCVD and solution-doping techniques with $\mathrm{Bi}\left(\mathrm{NO}_{3}\right)_{3}$ as a raw material. The fiber samples were singlemode for the pump light wavelength $(\sim 1 \mu \mathrm{m})$. Core radii $r_{0}$ of the drawn $\mathrm{Bi}$-doped fibers were in the range from 2.0 to $3.0 \mu \mathrm{m}$ and their core-field overlap factors $\Gamma$ were nearly the same, of $\sim 0.8$.

\section{A. Absorption spectra}

Typical optical loss (SSA) spectra of the tested Bi-doped fibers are shown in Fig. 1. The presented spectra were obtained using a white light source with a fiber output and an optical spectrum analyzer (OSA) with a $1 \mathrm{~nm}$ resolution. The

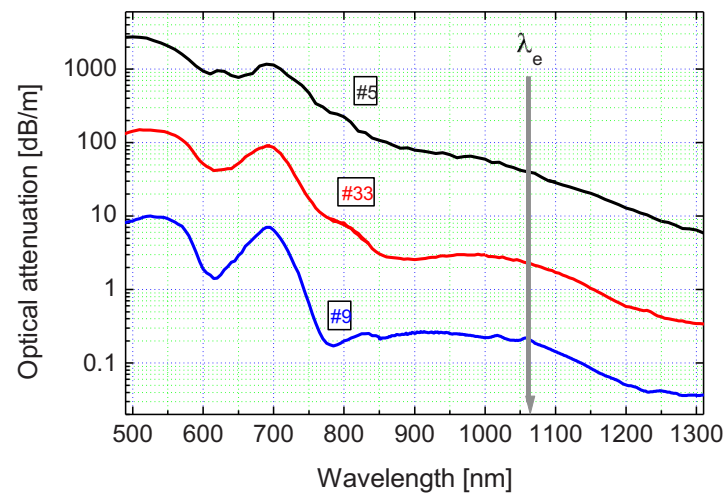

FIG. 1. (Color online) Attenuation (SSA) spectra of the Bi-doped fibers with low (sample no. 9), intermediate (sample no. 33), and high (sample no. 5) Bi centers' contents. 


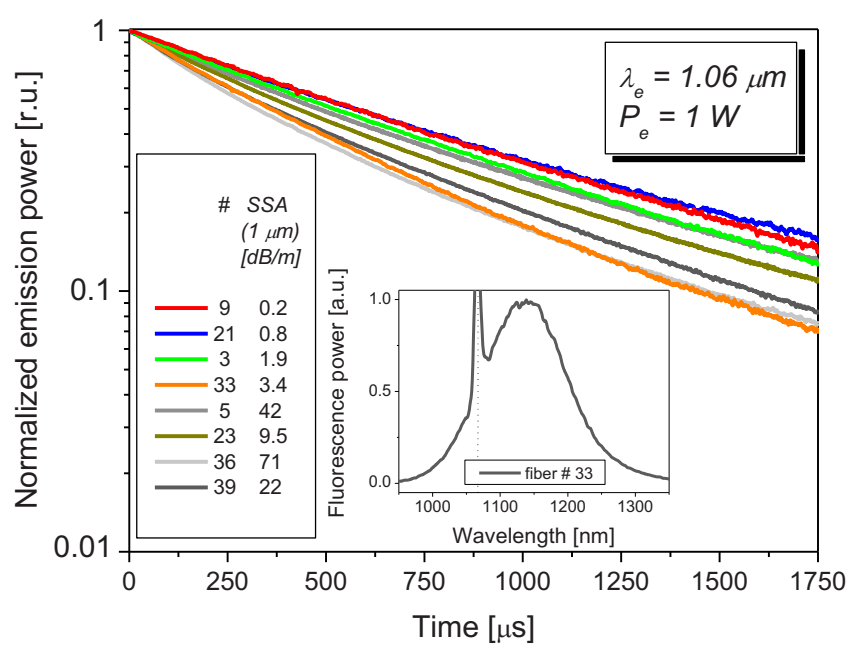

FIG. 2. (Color online) Normalized NIR fluorescence decays obtained for the Bi-doped fibers with different contents of Bi centers. Left-hand inset shows the attributions for the fiber samples, each labeled by the SSA (at $\lambda_{e}$ $=1.06 \mu \mathrm{m}$ ) value. Right-hand inset demonstrates an example of Bi centers' fluorescence spectrum at excitation at $\lambda_{e}=1.06 \mu \mathrm{m}$ (fiber no. 33).

pump wavelengths employed in our experiments fall approximately in the center of the absorption band 900-1200 nm of Bi centers.

The optical loss level of glass matrix estimated at $1.3 \mu \mathrm{m}$ was much smaller (at least an order of magnitude) than that within the absorption band, so we assume that the main reason of optical loss at $\sim 1 \mu \mathrm{m}$ wavelength is Bi centers absorption. It is seen that the SSA spectra have a very similar shape for all the fibers under study, differing only in intensity. Notice that NIR fluorescence of $\mathrm{Bi}$ centers being produced by $\sim 1 \mu \mathrm{m}$ excitation and centered at $\sim 1150 \mathrm{~nm}$ wavelength has also a quite similar shape for all fibers in the set. It is also noteworthy a virtually exponential growth of "background" loss toward shorter wavelengths in the attenuation spectra, which accompanies an increase in absorption peaks $(\sim 550, \sim 700$, and $\sim 1050 \mathrm{~nm})$ ascribed to active $\mathrm{Bi}$ centers, responsible for lasing. This background attenuation is undoubtedly associated with $\mathrm{Bi}$, but its nature is yet questionable.

\section{B. Fluorescence decay}

The kinetics of the fibers' NIR fluorescence was measured after excitation at $1064 \mathrm{~nm}$ wavelength using a bulk $\mathrm{CW}$ yttrium-aluminum garnet (YAG): $\mathrm{Nd}^{3+}$ laser; typical fluorescence decays are presented in Fig. 2. The laser was modulated by an electro-optical modulator to achieve squareshaped pulses of millisecond-width; so its output power $P_{e}$ was modulated between zero and $\sim 1 \mathrm{~W}$. To avoid possible reabsorption, Bi fluorescence was detected from the lateral surface of the fiber samples, using a Ge photodetector. The detector had almost plain quantum yield within a spectral interval $750-1600 \mathrm{~nm}$ so that optical power of $\mathrm{Bi}$ centers fluorescence peaked at $1150 \mathrm{~nm}$ was whole transformed in an electrical signal. The time resolution of our set-up was checked out in advance to experiments and was found to be $\sim 8 \mu$ s. So, all the dependences presented in Fig. 2 were obtained after normalization of the decay curves on the values of fluorescence signals recorded at $10 \mu$ s after the pump switching off.

The magnitude of a pump pulse $(\sim 1 \mathrm{~W})$ provided a full saturation of ground-state absorption (GSA), which takes place at $P_{e} \approx 0.2 \mathrm{~W}$. This allowed us to neglect in a subsequent analysis an intensity-dependent contribution in NIR fluorescence. $^{28}$ The knowledge of the fluorescence decays allowed us in turn to get for each Bi-doped fiber the lifetime $\tau_{0}$ and HUC coefficient $C_{\mathrm{UP}}$ and to build thereafter the dependences of $\tau_{0}$ and $C_{\mathrm{UP}}$ on the SSA value $\alpha_{0}$.

From Fig. 2, one can conclude that decays of NIR fluorescence of $\mathrm{Bi}$ centers is a function of the SSA value $\alpha_{0}$ (and so of Bi concentration, apparently proportional to SSA). The fibers with the smallest content of $\mathrm{Bi}$ centers $\left(\alpha_{0}\right.$ $<1 \mathrm{~dB} / \mathrm{m}$ ) demonstrate practically single-exponential fluorescence decay, whereas the decay kinetics gets more and more deviated from the exponential law with increasing $\alpha_{0}$. At the highest content of Bi centers $\left(\alpha_{0}>10 \mathrm{~dB} / \mathrm{m}\right)$, the decay kinetics becomes essentially nonexponential, being a result of the HUC process discussed in what follows.

We also found reasonable to show here a typical fluorescence spectrum of $\mathrm{Bi}$ centers at $1064 \mathrm{~nm}$ excitation: See inset to Fig. 2 where we plot the data for fiber no. 33 (fluorescence spectra of other fibers in the set have a very similar shape $^{25}$ ). One can reveal from the inset that $\mathrm{Bi}$ centers of alone type (but not multiple: attention should be paid to an almost symmetrical shape of the spectrum) is responsible for the fluorescence peaked at $1150 \mathrm{~nm}$, decay of which was discussed above.

\section{Transmission coefficient}

The dependence of the fibers samples' transmission on the signal power was measured with a CW YFL with $\lambda_{e}$ $=1056 \mathrm{~nm}$ wavelength. Output power of the YFL was varied from $\sim 1 \mathrm{~mW}$ to $\sim 1 \mathrm{~W}$. The tested fiber samples were spliced to the YFL output and their transmission coefficient was calculated as $T=P^{\text {out }} / P^{\text {in }}$, with $P^{\text {in }}$ and $P^{\text {out }}$ being the pump powers on the input and output ends of a fiber. The powers $P^{\text {in }}$ and $P^{\text {out }}$ were measured using a standard power meter. We also checked with the OSA, for each fiber, a ratio of the signal power and power of amplified spontaneous emission (ASE); ASE was found to be everywhere negligible.

The nonlinear transmission coefficient $T$ as a function of pump power is shown in Fig. 3 for the most representative fibers, having the lowest SSA values at $\sim 1 \mu \mathrm{m}$ wavelength $\left(\alpha_{0}<3.5 \mathrm{~dB} / \mathrm{m}\right.$, fiber nos. 9, 21, 3, and 33; see Table I). In this case, contrast between the unsaturated and saturated absorptions is the biggest. Furthermore, the fiber lengths $L$ were experimentally chosen for each fiber such that the highest contrast is provided within the available range of signal powers. The main parameters of these fibers are given in the Table I. Notice that the Bi-doped fibers with the highest $\alpha_{0}$ values $(>10 \mathrm{~dB} / \mathrm{m}$; e.g., fiber no. 5 whose SSA spectrum is presented in Fig. 1) demonstrate almost no bleaching.

The dependences $T\left(P^{\text {in }}\right)$ [Figs. 3(a)-3(d)] have a similar 

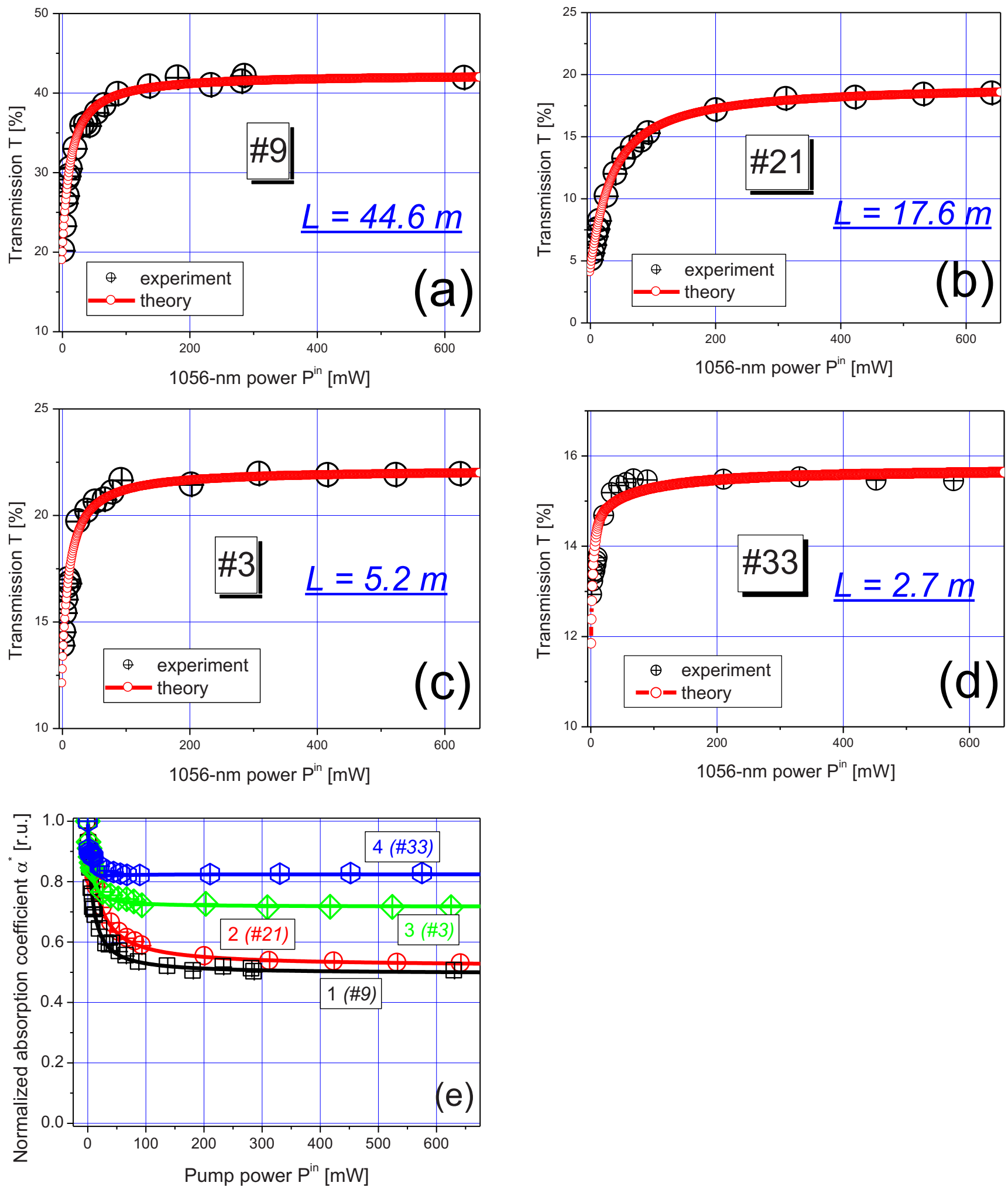

FIG. 3. (Color online) Coefficients of nonlinear transmission $T$ [(a)-(d)] and normalized absorption $\alpha^{*}$ (e) vs pump power $P^{\text {in }}$. Curves $1-4$ on figure (e) correspond to the curves plotted on figures [(a)-(d)], after recalculating transmission to normalized absorption. Symbols—experimental data; plain curvesresults of modeling.

nonlinear character, that is, a fast growth of transmission, starting from $P^{\text {in }} \sim 1 \mathrm{~mW}$ and getting saturated at $P^{\text {in }}$ in excess of $\sim 100 \mathrm{~mW}$. "Plateaus" in the transmission curves are observed at higher signal powers, $P^{\text {in }} \sim 0.5 \mathrm{~W}$; their levels are seen to be dependent on the Bi centers concentration (i.e., on the SSA value).
Another important feature can be revealed from Fig. 3(e), where we demonstrate the results of formal recalculation of the experimental data plotted on Figs. 3(a)-3(d). This recalculation turns the transmission coefficient $T\left(P^{\text {in }}\right)$ into the absorption coefficient, normalized on the SSA value; $\alpha^{*}\left(P^{\text {in }}\right)=-\left[\ln (T) / L \alpha_{0}\right]$. The degree of absorption bleaching 
TABLE I. Parameters of four fiber samples with the lowest Bi centers contents.

\begin{tabular}{|c|c|c|c|c|c|c|c|c|c|c|}
\hline No. & $\begin{array}{l}\text { Sample } \\
\text { length } \\
(\mathrm{cm})\end{array}$ & $\begin{array}{c}\text { Loss } \\
\text { @ } 1064 \mathrm{~nm} \\
(\mathrm{~dB} / \mathrm{m})\end{array}$ & $\begin{array}{c}\text { Grey loss } \\
\text { @ } 1270 \mathrm{~nm} \\
(\mathrm{~dB} / \mathrm{m})\end{array}$ & $\begin{array}{c}\text { Doped } \\
\text { core radius } \\
(\mu \mathrm{m})\end{array}$ & $\begin{array}{l}\text { Mode } \\
\text { field radius } \\
\qquad(\mu \mathrm{m})\end{array}$ & $\begin{array}{c}\text { Transmission } \\
\text { coefficient } \\
\text { (initial/final) } \\
(\%)\end{array}$ & $\begin{array}{c}k C_{\mathrm{UP}} \\
\left(\mathrm{s}^{-1}\right)\end{array}$ & $\begin{array}{c}\text { Pairs } \\
\text { relative weight } \\
\kappa \\
(\%)\end{array}$ & $\begin{array}{c}\text { Active } \\
\text { absorption } \\
(\mathrm{dB} / \mathrm{m})\end{array}$ & $\begin{array}{c}\text { NSA } \\
(\mathrm{dB} / \mathrm{m})\end{array}$ \\
\hline 9 & 4460 & 0.16 & 0.01 & 2.03 & 2.36 & $18.2 / 41.2$ & 45 & 0 & 0.07 & 0.08 \\
\hline 21 & 1755 & 0.82 & 0.03 & 2.17 & 2.52 & $4.1 / 18.8$ & 230 & 4 & 0.37 & 0.42 \\
\hline 3 & 520 & 1.79 & 0.06 & 2.04 & 2.71 & $12.3 / 22.1$ & 510 & 53 & 0.47 & 1.26 \\
\hline 33 & 275 & 3.63 & 0.26 & 2.43 & 2.83 & $12.0 / 15.4$ & 930 & 89 & 0.44 & 2.93 \\
\hline
\end{tabular}

steadily decreases through the set of the fibers, which is characterized by a raised SSA value (nos. 9, 21, 3, and 33). This behavior is clearly seen from a direct comparison of the plateaus' levels in Fig. 3(e).

The nonlinear character of the dependences of Bi-doped fibers' transmissions (absorptions) versus pump power (Fig. 3) stems from the GSA saturation and probably from the ESA and UC processes. Indeed, our attempts to fit these experimental dependences by an account of the GSA saturation only have been failed (due to a huge level of the nonsaturable loss). So, we have found imperative to take into consideration the ESA and UC processes in the analysis in an attempt to address the real situation with the fibers.

\section{MODELING}

We develop next a modeling for both the experiments with a set of the Bi-doped fibers, i.e., for the measured kinetics of NIR fluorescence (Fig. 2) and for the nonlinear transmission coefficient (Fig. 3). A proper account in the modeling of the GSA and ESA transitions and also of the interaction effects (appeared as HUC and IUC) allows us to get consistency between the experiment and theory and to find thereafter the values of a few important constants for the characterization of $\mathrm{Bi}$-doped alumino-silicate fibers.

The basic idea of our modeling is that ensemble of $\mathrm{Bi}$ centers consists of two subsystems, considered to be the ones of single and paired centers (the simplest clusters), similarly to the approach long-ago developed for the characterization of heavily doped erbium and ytterbium doped fibers. ${ }^{29-35}$ The modeling's logic is as follows:

(i) primary inspection of the kinetics of NIR fluorescence decays obtained for the entire set of the fiber samples with different Bi centers' contents; this allows us to obtain lifetimes $\tau_{0}$ and HUC coefficients $C_{\mathrm{UP}}$;

(ii) subsequent inspection of the nonlinear transmission coefficient of the Bi-doped fiber samples with SSA less than $3.5 \mathrm{~dB} / \mathrm{m}$, i.e., of the ones with nonsaturable absorption (NSA) yet not so pronounced to prevent lasing and amplification; ${ }^{25}$ the knowledge of the found constants $\tau_{0}$ and $C_{\mathrm{UP}}$ allows us to find the parameter characterizing the ESA to GSA ratio $(\eta)$ and the relative percentage of single and paired centers in the fibers.

\section{A. Fluorescence decay}

It is seen from Fig. 2 that the NIR fluorescence decay gets deviated from the single-exponent law, when the concentration of Bi centers increases (and so SSA does). For an erbium doped medium such a behavior is accepted to stem from HUC, i.e., from the energy transfer process between equivalent centers, both being in the excited state, with the result that one of these decays to the ground state and another, after rapid nonradiative relaxation, remains in the excited state. We believe that such a treatment is fully applicable in our case (single Bi centers). It is clear that this will result in a nonexponential character of the kinetics that may be also referred to as fluorescence concentration quenching.

For the normalized population density $n_{2}^{S}$ of single (index $s$ ) $\mathrm{Bi}$ centers in the excited state, the rate equation is written as follows:

$$
\frac{d n_{2}^{S}}{d t}=-\frac{n_{2}^{S}}{\tau_{0}}-C_{\mathrm{UP}}\left(n_{2}^{S}\right)^{2} .
$$

where $n_{2}^{S}=N_{2}^{S} / N_{0}^{S} ; N_{2}^{S}$ is the population density of single Bi centers in the exited state, $N_{0}^{S}$ is the centers concentration, and $C_{\mathrm{UP}}\left(\mathrm{s}^{-1}\right)$ is the UC parameter, being a product of standard "volumetric" HUC constant $C_{\mathrm{UP}}^{*}\left(\mathrm{~s}^{-1} \mathrm{~cm}^{3}\right)$ and $N_{0}^{S}$.

Let us assume that the excitation power is high enough to achieve a maximal population of the excited state. The population of this state is limited by the relation between GSA and stimulated emission (SE) cross-sections, $\sigma_{12}$ and $\sigma_{21}$. The part $k$ of $\mathrm{Bi}$ centers that can be transited in the excited state under pumping with "infinite" power is defined as $k=\sigma_{12} /\left(\sigma_{12}+\sigma_{21}\right)$. At our assumption, $n_{2}^{s}(t=0) \equiv k$; so we can obtain an analytical solution of Eq. (1), which describes the fluorescence decay after the pump light switching off as follows:

$$
n_{2}^{S}(t)=k \frac{e^{-t / \tau_{0}}}{1+k \tau_{0} C_{\mathrm{UP}}\left(1-e^{-t / \tau_{0}}\right)} .
$$

The derived formula is applicable for fitting the normalized NIR fluorescence decays obtained experimentally for the entire set of the Bi-doped fibers (Fig. 2), as for all these, having the same chemical host, the quantities $\tau_{0}, C_{\mathrm{UP}}$, and $k$ should be the same at the excitation at $\lambda_{e}$.

The results are demonstrated in Fig. 4 where plotted are the dependences of lifetime of single Bi centers $\tau_{0}$ and HUC parameter $k C_{\mathrm{UP}}$ against the SSA value $\alpha_{0}$. Note that in our case, $k \approx 0.5$ for $\lambda_{e} \approx 1.06 \mu \mathrm{m}^{2}$ 

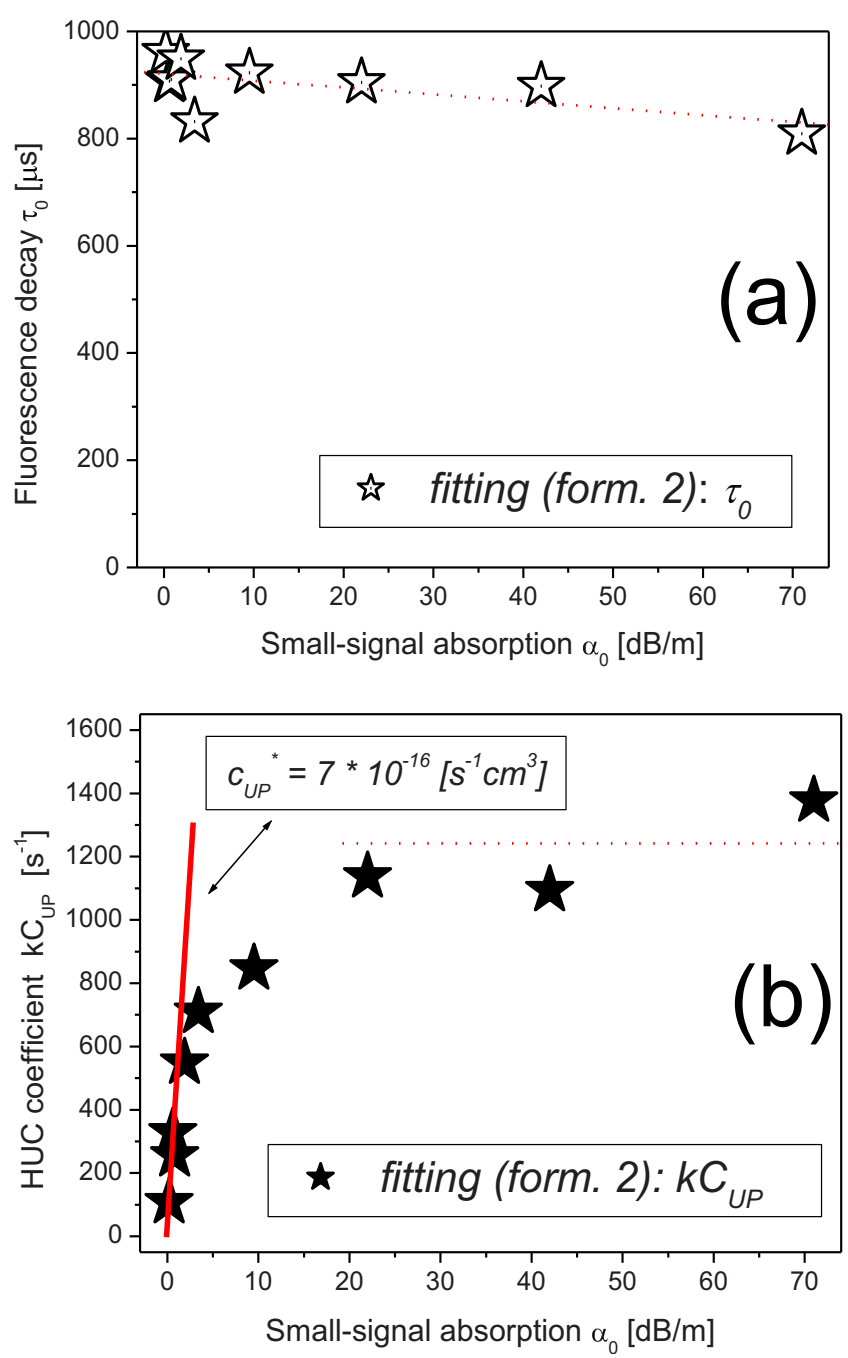

FIG. 4. (Color online) Dependences of NIR fluorescence decay time $\tau_{0}$ (a) and HUC constant $k C_{\mathrm{UP}}(\mathrm{b})$ on SSA value $\alpha_{0}$. The red line on figure (b) is a linear fit that defines the HUC constant value: $c_{\mathrm{UP}}^{*}=7 \times 10^{-16} \mathrm{~s}^{-1} \mathrm{~cm}^{3}$.

It is seen that $\tau_{0}$ is almost constant $(\sim 900 \mu \mathrm{s})$ through the whole range of SSA values (or concentrations of Bi centers), having only a slight trend to drop with increasing the $\mathrm{Bi}$ centers content. In the meantime, the HUC parameter $\left(k C_{\mathrm{UP}}\right)$ is a strong function of $\alpha_{0}$ (or $\left.N_{0}\right)$. Generally to say, the dependence $k C_{\mathrm{UP}}\left(N_{0}\right)$ is expected to be a linearly increasing function and it is indeed the case for smaller $\alpha_{0}$, that span from zero to several decibels per meter [see the red fit in Fig. 4(b)]. For larger $\alpha_{0}$, i.e., for the higher overall concentrations of $\mathrm{Bi}$ centers, the growth of $k C_{\mathrm{UP}}$ gets saturated. This behavior is originated from the fact that $C_{\mathrm{UP}}$ is proportional to $N_{0}^{S}$, but not to $N_{0}$, because the HUC process is an attribute of single centers (on the contrast, paired centers experience IUC, see below). So, $k C_{\mathrm{UP}}$ is proportional to a part of $\alpha_{0}$, which becomes smaller with an increase in $\mathrm{Bi}$ centers concentration. The last point will be confirmed below by the results of a modeling of the nonlinear transmission coefficient of the Bi-doped fibers.

The HUC process figured out above has a deep physical relation to the ESA one; namely, the $\mathrm{HUC}$ constant $C_{\mathrm{UP}}^{*}$ is proportional to the ESA cross-section. ${ }^{36}$ The next results on the modeling of the nonlinear transmission coefficient justify an important role of the ESA transition in the Bi-doped alumino-silicate fibers. The value of the HUC constant, $C_{\mathrm{UP}}^{*} \equiv C_{\mathrm{UP}} / N_{0}^{S} \approx 7 \times 10^{-16} \mathrm{~s}^{-1} \mathrm{~cm}^{3}$, was obtained for the case of low $\mathrm{Bi}$ centers concentrations $N_{0}^{S} \approx N_{0}$; notice that this value matches by the order of magnitude to an estimate recently made from the experiments on fluorescence saturation in Bi-doped fibers. ${ }^{26}$ Needless to say, a strong spectral dependence of the HUC parameter $k C_{\mathrm{UP}}$ is expected since it is a function of the excitation wavelength $\lambda_{e}$; this matter should be accounted for at further studies with Bi-doped fibers.

\section{B. Transmission coefficient}

To model the behavior of nonlinear transmission (or absorption) versus signal power (see Fig. 3), we have derived a system of equations that address the propagation of a signal at the wavelength $\lambda_{e}$ through a Bi-doped fiber sample. This was done under the assumption that there are two subsystems of Bi centers, single $(s)$ and paired $(p)$ ones (we believe that aggregates of closely situated $\mathrm{Bi}$ centers are formally considered as "pairs," at least in the first approximation).

A simplified attribution for each kind of the centers' groups and for the processes involved at $\sim 1 \mu \mathrm{m}$ pumping is implied. That is, we model the complex real situation by a simplified system with three levels, where level 1 is the ground state, level 2 is the first excited state, and level 3 is a higher lying excited state (this last in fact reflects a possible presence of many higher-lying excited states including the virtual ones). Pump photon energy is considered to correspond to the separation between levels 1 and 2 while direct transitions between levels 1 and 3 are considered to be not allowed since the separation between them is larger than the photon energy.

Under these assumptions, we formulate the following rules which the subsystems of single and paired Bi centers obey:

(i) Single centers. They can occupy the ground state "1" and the first excited state "2." Migration between these states is driven by pumping (GSA and SE) and spontaneous emission. Transition to the state " 3 " is possible only from the state " 2 " through the ESA and HUC processes. The ESA cross-section is defined as $\sigma_{23}$ (the GSA and SE cross-sections have been defined above as $\sigma_{12}$ and $\sigma_{21}$, respectively). ESA involves only one center but HUC requires an interaction between two single centers both being in the excited state "2." In the result of the latter, one of the centers turns in the state "1" and the other goes up in the state "3." It is implied that the center being in the state " 3 " instantaneously returns to the state " 2 " $\left(\tau_{32} \approx 0\right)$, so there is no change in the population of the state " 2 " and the population of the state " 3 " is always zero.

(ii) Paired centers. Not looking deeply inside the physical phenomenon of IUC, we postulate here that the only difference compared to the previous case is that the centers could not be in the excited state in the same time. Once they are, one of them immediately goes down to the ground state, and the other goes up to the 
state, physical nature of which is a new state of the pair, or virtual state not observed at the measurements of SSA. Namely, such a situation is called IUC. We assume further that the state where both the centers are excited is quickly depopulated and its population can be zeroed. So, the pair as a whole has only the two states, state $\langle 11\rangle$ (when both centers are in the ground state) and state $\langle 12\rangle$ (when only one of them is in the excited state). Evidently, HUC is not applicable to the paired centers, and vice-versa IUC is not applicable to the single centers. The processes in paired centers arising under the action of pump light are assumed to be characterized by the same quantities as those introduced above for singles $\left(\sigma_{12}, \sigma_{21}, \sigma_{23}\right.$, and $\left.\tau_{0}\right) .^{37}$ Of worth noticing should be one of the IUC manifestations, the NSA loss, which originates from the fact that one of the pair' constituents always occupies the ground state, irrespectively to the pump power.

We assume in what follows that the population densities of single and paired centers $N_{1,2}^{s, p}$ satisfy the following relations: $N_{1}^{s}+N_{2}^{s}=N_{0}^{s}=N_{0}(1-2 \kappa) ; N_{1}^{p}+N_{2}^{p}=N_{0}^{p}=2 \kappa N_{0}$, where $\kappa$ is the partial weight of paired centers in the ensemble and $N_{0}$ is the overall $\mathrm{Bi}$ centers concentration. Their normalized counterparts are defined as $n_{1,2}^{s, p}=N_{1,2}^{s, p} / N_{0}$; lower indices here and further attribute, correspondingly, the ground (" 1 " or $\langle 11\rangle$ ) and first excited (" 2 " or $\langle 12\rangle$ ) states, and $N_{0}^{S, P}$ are the concentrations of single and paired Bi centers, respectively.

In this case, the balance equations for the pump power $P$ and for the normalized dimensionless population densities of paired and single centers in the meta-stable states " 2 " and $\langle 12\rangle n_{2}^{s, p}\left(0 \leq n_{2}^{s, p} \leq 1\right)$ are (see, e.g., Refs. 29, 32, and 33) as follows:

$$
\begin{aligned}
& \frac{d P}{d z}=-\alpha_{0} P\left\{1-(\xi-\eta)\left(n_{2}^{S}+n_{2}^{P}\right)\right\}-\gamma_{0} P, \\
& \frac{\sigma_{12} \Gamma}{h \nu S_{a}}\left[2 \kappa-(1+\xi) n_{2}^{P}\right] P-\frac{n_{2}^{P}}{\tau_{0}}=0, \\
& \frac{\sigma_{12} \Gamma}{h \nu S_{a}}\left[1-2 \kappa-\xi n_{2}^{S}\right] P-\frac{n_{2}^{S}}{\tau_{0}}-C_{\mathrm{UP}}\left(n_{2}^{S}\right)^{2}=0,
\end{aligned}
$$

where quantities $\alpha_{0}, \Gamma, \tau_{0}$, and $C_{\mathrm{UP}}$ have been defined above; $\xi=\left(\sigma_{12}+\sigma_{21}\right) / \sigma_{12} \equiv 1 / k$ and $\eta=\sigma_{23} / \sigma_{12}$ are the coefficients that stand for the ratios between the GSA, ESA, and SE cross-sections; $\gamma_{0}$ is the linear loss coefficient; $S_{a}$ is the geometrical cross-section of Bi-doped core; $h \nu=h c / \lambda_{e}$ is the pump energy quanta. We neglect in Eqs. (3)-(5) the ASE contribution as it was negligible in the experiments.

We calculated output power $P^{\text {out }}$ as a function of $P^{\text {in }}$ for each sample and then calculated the transmission coefficient as $T=P^{\text {out }} / P^{\text {in }}$. So, the modeling results are directly comparable with the experimental data (see Fig. 3). Numerical calculations were processed for the fibers with moderate SSA values (see the Table I).

Majority of the parameters' values employed in the modeling were obtained from the experiments (see the Table I), apart from the constants characterizing the NIR fluorescence decay, obtained above $\left(\tau_{0}=920 \mu \mathrm{s}\right.$ and $C_{\mathrm{UP}}^{*}=7$ $\times 10^{-16} \mathrm{~s}^{-1} \mathrm{~cm}^{3}$; see Fig. 4). The coefficient characterizing the GSA to SE ratio was determined from the data published in Ref. $2 ; \xi=1.48$ (for all the fibers). The pump intensity that saturates the GSA transition was taken as $I_{s}=50 \mathrm{~kW} / \mathrm{cm}^{2}$ (this value corresponds to the saturating pump power, $\sim 100 \mathrm{~mW}$; see Fig. 3 and the Table I). The GSA crosssection was formally calculated as follows: $\sigma_{12}=h \nu /\left(I_{s} \tau_{0} \xi\right)$ $\left(\approx 2.7 \times 10^{-21} \mathrm{~cm}^{2}\right)$. The overall concentrations of Bi centers $\left(N_{0}\right)$ in the fibers was estimated using the following relation: $N_{0}=\alpha_{0} /\left(\Gamma \sigma_{12}\right)$. The linear loss coefficient $\gamma_{0}$ was accepted to stem from the background loss measured at the wavelength $1270 \mathrm{~nm}$ (see Fig. 1 and the Table I). The ESA to GSA ratio $(\eta=0.72)$ was determined for the fiber with the smallest SSA value (no. 9, for which we assume no contribution from paired centers; $\kappa=0$ ) and it was further kept unchanged for the rest of the fibers, heavier doped with $\mathrm{Bi}$ (because the ESA cross-section is apparently not concentrationdependent). It is worthy to notice that a unique parameter used to fit the experimental dependences shown in Fig. 3 was the coefficient $\kappa$ (the relative "weight" of paired centers in the fibers nos. 21, 3, and 33).

The results of numerical calculations of Eqs. (3)-(5) are presented by plain curves in Fig. 3. It is seen that the theoretical curves quantitatively fit the experimental dependences (empty symbols) for all the fibers under study. Taking into account the results on fluorescence decays' modeling (see above), we conclude that the ESA and UC processes are proven to be responsible for the presence in the fibers of the NSA loss.

Another result of our modeling, which stems from the best fit of the experiments data by the developed theory, is that we can plot now the relative weights of paired and single Bi centers against the SSA value, $\alpha_{0}$ [see Fig. 5(a)] and also the dependences on SSA of "active" (bleachable) absorption $\alpha_{a}$ and "passive" (NSA) loss $\alpha_{r}$ [see Fig. 5(b)], the quantities being defined by the presence in the fibers of mainly single and mainly paired centers, correspondingly.

The presented results have been obtained for the simplest IUC theory where the most natural case of Bi centers clustering ("pairing") is treated. We believe that this approach is satisfactory for the fibers with relatively low $\mathrm{Bi}$ centers content (i.e., with low SSA, not exceeding several decibels per meter). Meanwhile, a similar theory can be developed to cover the case of more complicate Bi clusters, applicable for heavier doped fibers (like it has been done for rare-earth doped fibers ${ }^{29-37}$ ).

Let us evaluate now a critical interaction distance between $\mathrm{Bi}$ centers, $R_{0}^{\mathrm{HUC}}$, the parameter, characterizing strength of the HUC process in the fibers, as follows:

$$
R_{0}^{\mathrm{HUC}}=\sqrt[6]{\frac{9 C_{\mathrm{UP}}^{*} h \nu \Gamma}{16 \pi^{2} \xi I_{s} \alpha_{0}}} .
$$

Notice that formula (6) is obtained at the assumptions of (i) a linear dependence of HUC coefficient on concentration of single $\mathrm{Bi}$ centers $N_{0}^{S}$ (valid for SSA coefficients $\alpha_{0}$ $<3.5 \mathrm{~dB} / \mathrm{m}$ ), (ii) dipole-dipole interaction between Bi cen- 

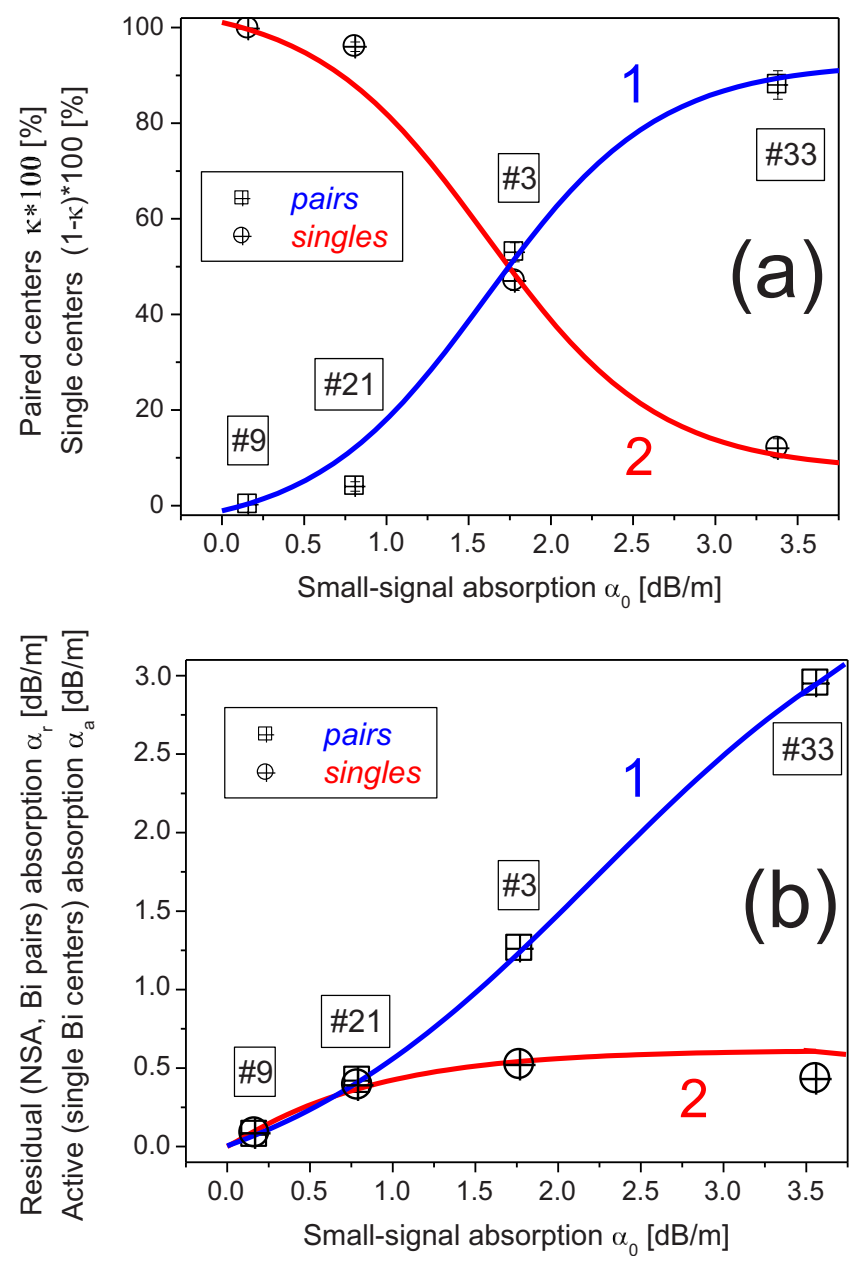

FIG. 5. (Color online) Relative "weights" of paired and single Bi centers (a) and the correspondent NSA and "active" absorption coefficients of the Bidoped fibers vs SSA.

ters at HUC, and (iii) negligible IUC. ${ }^{37}$ The results of calculations using formula (6) reveal that $R_{0}^{\mathrm{HUC}}$ for the host glass of our fibers is nearby $1.5-2 \mathrm{~nm}$, being a weakly decreasing function of SSA and concentration of Bi centers. This result, found for Bi centers, is seen to be akin of those for rare-earth doped silica glass.

Let us make also an estimation-needless to say, rough-for the distance between Bi centers that form a pair (a "chemical cluster" responsible for the IUC process). Since this distance is usually estimated to be a half-order less than $R_{0}^{\mathrm{HUC}, 35}$ it should be measured in our case by units of angstrom.

Before to conclude, let us stress again that attribution of emission-active Bi centers in silica fibers is yet questionable; see, e.g., Refs. 8-19, 38, and 39. For instance, there are some evidences ${ }^{38,39}$ for the existence of a few kinds of active centers associated with the presence of $\mathrm{Bi}$ in silica glass. Nonetheless we have demonstrated above that, in the sense of the nonlinear absorption and NIR fluorescence kinetics at $\sim 1 \mu \mathrm{m}$ excitation, an assumption of alone kind of Bi dopants (single or paired centers) in alumino-silicate fiber is quite satisfactory.

\section{CONCLUSIONS}

We have experimentally studied the nonlinear transmission coefficient and the kinetics of NIR fluorescence decays for a series of Bi-doped alumino-silicate fibers. It was recognized that the ESA and UC processes stand behind a growth of NSA and fluorescence quenching at increasing Bi centers concentration in the fibers. This has been demonstrated by means of a modeling that implies Bi dopants being ensemble composed of single and paired centers with an increase in impact of the latter at a growth of Bi centers concentration. Thereafter, some important constants characterizing the ESA, HUC, and IUC processes have been found. In particular, the ESA coefficient, i.e., the ratio of ESA to GSA crosssection, has been found to be 0.72 at $\sim 1.06 \mu \mathrm{m}$ wavelength and the HUC constant to be around $7 \times 10^{-16} \mathrm{~s}^{-1} \mathrm{~cm}^{3}$.

\section{ACKNOWLEDGMENTS}

The authors thank Dr. A. A. Umnikov and Dr. A. N. Guryanov (Institute for Chemistry of High-Purity Substances of the Russian Academy of Sciences, Nizhny Novgorod, Russia) for the fabrication of the Bi-doped alumino-silicate fibers and Dr. N. N. Il'ichev (A. M. Prokhorov General Physics Institute, Moscow, Russia) for the useful discussions.

${ }^{1}$ M. Peng, J. Qiu, D. Chen, X. Meng, I. Yang, X. Jiang, and C. Zhu, Opt. Lett. 29, 1998 (2004).

${ }^{2}$ E. M. Dianov, V. V. Dvoyrin, V. M. Mashinsky, A. A. Umnikov, M. V. Yashkov, and A. N. Guryanov, Quantum Electron. 35, 1083 (2005).

${ }^{3}$ M. Peng, J. Qiu, D. Chen, X. Meng, and C. Zhu, Opt. Lett. 30, 2433 (2005).

${ }^{4}$ V. V. Dvoyrin, V. M. Mashinsky, L. I. Bulatov, I. A. Bufetov, A. V. Shubin, M. A. Mel'kumov, E. F. Kustov, E. M. Dianov, A. A. Umnikov, V. F. Khopin, M. V. Yashkov, and A. N. Guryanov, Opt. Lett. 31, 2966 (2006).

${ }^{5}$ E. M. Dianov, A. V. Shubin, M. A. Mel'kumov, O. I. Medvedkov, and I. A. Bufetov, J. Opt. Soc. Am. B 24, 1749 (2007).

${ }^{6}$ A. B. Rulkov, A. A. Ferin, S. V. Popov, J. R. Taylor, I. Razdobreev, L. Bigot, and G. Bouwmans, Opt. Express 15, 5473 (2007).

${ }^{7}$ V. V. Dvoyrin, V. M. Mashinsky, and E. M. Dianov, Opt. Lett. 32, 451 (2007).

${ }^{8}$ Y. Fujimoto and M. Nakatsuka, Jpn. J. Appl. Phys., Part 2 40, L279 (2001).

${ }^{9}$ Q. Qian, Q. Y. Zhang, G. F. Yang, Z. M. Yang, and Z. H. Jiang, J. Appl. Phys. 104, 043518 (2008).

${ }^{10}$ X. Meng, J. Qiu, M. Peng, D. Chen, Q. Zhao, X. Jiang, and C. Zhu, Opt. Express 13, 1628 (2005)

${ }^{11}$ M. Y. Sharonov, A. B. Bykov, and R. R. Alfano, J. Opt. Soc. Am. B 26, 1435 (2009).

${ }^{12}$ M. Peng, C. Zollfrank, and L. Wondraczek, J. Phys.: Condens. Matter 21, 285106 (2009)

${ }^{13}$ V. O. Sokolov, V. G. Plotnichenko, and E. M. Dianov, Opt. Lett. 33, 1488 (2008).

${ }^{14}$ E. F. Kustov, L. I. Bulatov, V. V. Dvoyrin, and V. M. Mashinsky, Opt. Lett. 34, 1549 (2009).

${ }^{15}$ B. Denker, B. Galagan, V. Osiko, I. Shulman, S. Sverchkov, and E. Dianov, Appl. Phys. B: Lasers Opt. 98, 455 (2010).

${ }^{16}$ M. A. Hughes, T. Suzuki, and Y. Ohishi, Opt. Mater. (Amsterdam, Neth.) 32, 368 (2009).

${ }^{17}$ E. F. Kustov, L. I. Bulatov, V. V. Dvoyrin, V. M. Mashinsky, and E. M. Dianov, J. Phys. B 43, 025402 (2010).

${ }^{18}$ Y. Fujimoto, J. Am. Ceram. Soc. 93, 581 (2010).

${ }^{19}$ E. M. Dianov, Quantum Electron. 40, 283 (2010).

${ }^{20}$ I. Razdobreev, L. Bigot, V. Pureor, A. Favre, G. Bouwmans, and M. Douay, Appl. Phys. Lett. 90, 031103 (2007).

${ }^{21}$ V. V. Dvoyrin, V. M. Mashinsky, and E. M. Dianov, IEEE J. Quantum Electron. 44, 834 (2008).

${ }^{22}$ M. P. Kalita, S. Yoo, and J. K. Sahu, Appl. Opt. 48, G83 (2009).

${ }^{23}$ M. P. Kalita, S. Yoo, and J. Sahu, Opt. Express 16, 21032 (2008).

${ }^{24}$ S. Yoo, M. Kalita, J. Nilsson, and J. Sahu, Opt. Lett. 34, 530 (2009).

${ }^{25}$ V. V. Dvoyrin, A. V. Kir'yanov, V. M. Mashinsky, O. I. Medvedkov, A. A. Umnikov, A. N. Guryanov, and E. M. Dianov, IEEE J. Quantum Electron. 
46, 182 (2010).

${ }^{26}$ I. A. Bufetov, S. V. Firstov, V. F. Khopin, A. N. Guryanov, and E. M. Dianov, Proceedings of the 34th European Conference on Optical Communication, Brussels, Belgium, 2008, Vol. 2, pp. 85-86.

${ }^{27}$ Y.-Q. Qui and Y.-H. Shen, Chin. Phys. Lett. 25, 2527 (2008).

${ }^{28}$ S. A. Payne, G. D. Wilke, L. K. Smith, and W. F. Krupke, Opt. Commun. 111, 263 (1994).

${ }^{29}$ J. Nilsson, B. Jaskorzynska, and P. Blixt, IEEE Photonics Technol. Lett. 5, 1427 (1993).

${ }^{30}$ R. S. Quimby, W. J. Miniscalco, and B. Thompson, J. Appl. Phys. 76, 4472 (1994).

${ }^{31}$ M. K. Davis, M. J. F. Digonnet, and R. H. Pantell, J. Lightwave Technol. 13, 120 (1995).

${ }^{32}$ C. Berkdemir and S. Ozsoy, J. Lightwave Technol. 27, 4642 (2009).
${ }^{33}$ X. Dong, P. Shum, N. Q. Ngo, H.-Y. Tam, and X. Dong, J. Lightwave Technol. 23, 1334 (2005).

${ }^{34}$ A. V. Kir'yanov, Y. O. Barmenkov, and N. N. Il'ichev, Opt. Express 13, 8498 (2005).

${ }^{35}$ F. Auzel and P. Goldner, Opt. Mater. (Amsterdam, Neth.) 16, 93 (2001).

${ }^{36}$ M. P. Hehlen, N. J. Cocroft, T. R. Gosnell, A. J. Bruce, G. Nykolak, and J. Shmulovich, Opt. Lett. 22, 772 (1997).

${ }^{37}$ P. Myslinski, D. Nguyen, and J. Chrostowski, J. Lightwave Technol. 15, 112 (1997).

${ }^{38}$ L. I. Bulatov, V. M. Mashinsky, V. V. Dvoyrin, E. F. Kustov, and E. M. Dianov, Quantum Electron. 40, 153 (2010).

${ }^{39}$ I. Razdobreev, H. El Hamzaoui, V. Y. Ivanov, E. F. Kustov, B. Capoen, and M. Bouazaoui, Opt. Lett. 35, 1341 (2010). 